\title{
The lived experience of expatriate nurses providing end of life care to Muslim patients in a
}

\section{Muslim country: An integrated review of the literature}

\section{Abstract}

Background: The provision of appropriate end of life care for patients who have different life experiences, beliefs, value systems, religions, languages, and notions of healthcare, can be difficult and stressful for the nurse. To date, research has focused predominately on nurses' experiences of end of life care for the Muslim patient who is an immigrant in another country.

Objectives: To critically review the literature related to the lived experiences of non-Muslim expatriate nurses providing end of life care for Muslim patients in their home country. Design: Integrative Literature Review

Data Sources: Comprehensive online search of Library Databases: Ovid, CINAHL, EBSCOHost; MEDLINE; Science Citation Index Expanded; PubMED; Web of Science; PROQUEST, and Scopus.

Review Methods: An integrative review of literature published within the dates January 2000 - July 2017. Included articles were published in the English language, peer reviewed/refereed, and focused on nurses' experiences. Both qualitative and mixed method studies describing the experience of non-Muslim nurses providing nursing care to Muslim patients in a country that was predominately Muslim were included.

Results: Initially 74 articles were found of which nine met the inclusion criteria. Research has been conducted predominantly within the Kingdom of Saudi Arabia, with one article from Bahrain and one other jointly from Kingdom of Saudi Arabia and the United Arab Emirates. The research indicates that expatriate nurses view themselves as powerless patient advocates, are hindered by the nurse-patient-family-physician quadriad structure, language and differing beliefs about communicating death, and negotiating culturally safe care is 
emotionally challenging.

Conclusion: This review highlights that the stressors associated with misalignment of expectations cause emotional and physical distress for the nurses. When nurses were focused on clinical care, they were unable to accommodate cultural practices that were important to the patient and family, contributing to increasing stress. Researchers have sought to capture this distress and make some sense of its impact. How nurses can provide culturally safe care, in countries with cultural practices quite different from their own, bears further investigation.

Keywords: cultural care; end of life care; Islam; Muslim; Muslim patient; nursing care 


\section{What is already known?}

- Lack of attention to specific cultural mores and beliefs may contribute to negative patient and family experiences of end of life care for Muslim migrants in Western cultures.

- Lack of knowledge of cultures limits nurses' abilities to practice culturally competent care.

- Expatriate non-Muslim nurses in a Muslim country can experience unique challenges in providing culturally competent care.

\section{What this paper adds?}

- For expatriate nurses working in a Muslim country, the differing language, culture and ways of communicating death can be emotionally demanding.

- While each is highly valued, the nurses perceive the relationships between the physician, patient, and family as a source of stress.

- The stress of managing the cultural differences and relationships can sometimes impact on nurses' ability to deliver end of life care. 


\section{Introduction}

Death and dying are personal and often spiritual events. Care of people nearing the end of life is a fundamental nursing role, where forming a therapeutic relationship with the patient and their family is critical. The culture, faith and personal beliefs of the person nearing the end of life is a paramount consideration for the nurse (Leininger, 1988). However, this work also carries the risk of stress and burnout (Halligan, 2006; Peters et al., 2013; Portoghese et al., 2014). Caring for people with different beliefs, values, religion, language, and expectation of healthcare adds complexities that can be demanding and stressful.

In the Middle East region, several countries have undergone rapid changes, moving from a nomadic culture to one that has the infrastructure and healthcare systems consistent with those in the western world (Al-Yateem et al., 2015; Hawkins, 2015). The Kingdom of Saudi Arabia, Bahrain, Qatar and United Arab Emirates rely heavily on expatriate nurses to fulfil their workforce (Aboshaiqah, 2016; El-Haddad, 2006). The reliance on a nursing workforce from abroad, often from quite different cultures, creates a unique set of concerns and issues in the provision of end of life care in the hospital setting (Abudari et al., 2016; Almutairi \& McCarthy, 2012; Halligan, 2006).

\section{Background}

Providing care and support for people who are approaching end of life, within the context of family, has been part of human social fabric since the beginning of time (Abel \& Kellehear, 2016; Reinhard et al., 2008). In the western world, end of life care has emerged as a specialty within the health industry, with the establishment of the discipline of palliative care in the 1960s bringing its own unique clinical outcomes, performance indicators and clinical competencies (Abel \& Kellehear, 2016; Ashby, 2016; Commonwealth of Australia, 2010; Eagar et al., 2010).

Discussing death and the dying process with patients and their families requires excellent communication skills and a thorough knowledge of physical and psychological changes common in the final days of life (Fairbrother \& Paice, 2005; Peterson et al., 2010). Good communication skills 
are recognised as a core competency in healthcare professionals. But even with good communication skills, nurses who care for people in the final stages of life experience various challenges in providing what is perceived as a "good death" (Peterson et al., 2010).

Nurses speak with, have eye contact with, and touch patients, often in very intimate ways near end of life. This intimacy places nurses at greater risk of moral distress when life or death situations evolve in disconcerting ways (Hanna, 2004). When there are cultural or language barriers, the stress associated with providing a good death can be further elevated (Cashavelly et al., 2008; Garner et al., 2013). When the stress is continuous, nurses may experience physical and emotional exhaustion or burnout (Ribeiro et al., 2014; Wu et al., 2011). Care at the end of life is often complex, with nurses managing multiple inter-related symptoms associated with terminal illness, engaging with distressed patients and families, and reflecting on suffering and death (Dréano-Hartz et al., 2015).

For the non-Muslim expatriate nurse working in a Muslim country; physical, psychological, spiritual, and cultural safety issues for the nurse, patients, families, and their communities often arise due to misunderstandings in communication and behaviours (Aboshaiqah et al., 2017; Almutairi \& Rodney, 2013; El-Amouri \& O'Neill, 2011). The challenges for nurses include high levels of stress (Halligan, 2006), and for those with no prior experience of Muslim patients, cultural shock can be expected (Al-Yateem et al., 2015). Nurses who are stressed and suffering physically and psychologically from culture shock may develop negative attitudes and feelings toward life and their clinical work, which in turn could affect their caregiving abilities and the quality of care delivered to patients (Lu et al., 2011; Peterson et al., 2010; Portoghese et al., 2014; Thurston \& Waterworth, 2012).

There is a growing body of literature focusing on caring for the Muslim patient and family in another country, where the Muslim patient is an immigrant and is being cared for by nurses in the nurses' home country (Bloomer \& Al-Mutair, 2013; Gatrad \& Sheikh, 2002; Salman \& Zoucha, 
2010; Somerville, 2007). The Muslim world comprises of a diversity of cultures and ethnicity.

There is a variety of religious practices across sects and non-Muslims are cautioned about believing there is homogeneity (Greenberg \& Haddonfield, 2007). For the purpose of this review, the collective term Muslim includes all followers of Islam.

As palliative care becomes an accepted specialty in the middle east health systems, more studies of how end of life care is provided to the Muslim patient in a Muslim country are emerging. The purpose of this review was to explore and critique the evidence regarding the experiences of non-Muslim expatriate nurses caring for patients near end of life in predominately Muslim countries.

\section{Design}

\subsection{The Review}

The review question was: What is the current evidence about the experiences and interactions of expatriate nurses providing end of life care to Muslim patients in a Muslim country? The objectives of the review included:

1. Identify published research studies focused on expatriate nurses caring for Muslim patients receiving end of life care;

2. Conduct a quality appraisal of the evidence in these studies; and

3. Identify themes from the findings.

\subsection{Design}

We selected an integrative review format to be inclusive of all research designs, including qualitative and mixed methods studies. An integrative review of evidence includes identification of the problem, literature search, data evaluation and analysis, which includes verification of data and drawing conclusions based on that analysis (Whittemore \& Knafl, 2005). Integrative reviews are useful when the scope of the topic is complex and uncertain (Bucknall et al., 2016), as they are the broadest type of research review, allowing for the simultaneous inclusion of experimental and non- 
experimental research in order to more fully understand a phenomenon of concern (Whittemore \& Knafl, 2005).

\subsection{Search strategy}

Databases searched included Ovid, CINAHL, EBSCOHost; MEDLINE; Science Citation Index Expanded; PubMED; Web of Science; PROQUEST, and Scopus in addition, the web and Google Scholar. We conducted a secondary search from reference lists and studies known to be relevant. The search was undertaken during April - September 2017. Inclusion criteria were: English language; published between 1 January 2000 - 31 July 2017 to obtain contemporary research; and focus on the Muslim patient being cared for in their home country. The reviewed literature included journal articles, doctoral thesis and books which represent the main texts in the relevant subjects. Key search terms were: nursing, Muslim patients, end of life, palliative care, and cultural care. The Preferred Reporting Items for Systematic Reviews and Meta-analyses, known as the PRISMA statement, was used to guide study selection (Liberati et al., 2009; Moher et al., 2009).

The author (SO) conducted the initial search and collated the search terms, databases and retrieved articles on a standardised data collection form, in the form of an Excel ${ }^{\circledR}$ spreadsheet. The initial online literature search yielded 74 results. The articles and spreadsheet were shared with two of the authors (LG, EC) for abstracts review. Following a review of abstracts, 15 studies met the inclusion criteria and these articles were submitted to data extraction and analysis. See Figure 1, outlining the PRISMA flowchart, for how relevant studies were identified.

$<$ Insert Figure 1 here $>$

\subsection{Data extraction}

We extracted data from the articles entered findings into an Excel® spreadsheet under the headings: author and year of publication, country, research aims and methods, setting and participants, key findings and conclusions. 
We assessed the quality of the studies using the Mixed Methods Appraisal Tool - Version 1 (Pluye \& Hong, 2014), with authors agreeing on the score following review of the articles. The benefits of this appraisal tool is that it can be used to assess all research designs, through the use of a quality scoring system of $0 \%, 25 \%, 50 \%$ or $100 \%$ with the higher score indicating higher quality (Pluye et al., 2009; Pluye \& Hong, 2014).

\subsection{Data Analysis}

Articles were read and reread aiming to avoid erroneous conclusions. Two authors (SO, LG) extracted key findings individually. Data were then shared and discussed over two meetings to confirm agreement. A third author (EC) reviewed cases where there was disagreement and provided a final decision. Through this process, the data were ordered, coded and categorised, grouped into themes and summarised (Polit \& Beck, 2017; Whittemore \& Knafl, 2005). The author (SA) contributed to the understanding of the cultural context and provided a critique of the thematic expression until all four authors agreed on final themes.

\section{Search outcome}

Seven studies were conducted in the Kingdom of Saudi Arabi (Abu-Ghori et al., 2016; Abudari et al., 2016; Abudari et al., 2014; Alasiry et al., 2012; Alosaimi \& Ahmad, 2016; Alosaimi et al., 2013; Halligan, 2006), one combined from the Kingdom of Saudi Arabia and the United Arab Emirates (AlYateem \& Al-Yateem, 2014) and one from Bahrain (O'Neill et al., 2017). Six studies used qualitative methods and one used a descriptive cross-sectional design (Abudari et al., 2014). A summary of the studies can be found in Table 1. Studies from the Kingdom of Saudi Arabia investigated nurses working in medical and surgical critical care, intensive care units, palliative care, oncology, medical, obstetrics, and gynaecology units. The study from Bahrain was of ten nurses working in intensive care units from two different hospitals, and the study of 27 nurses from the United Arab Emirates and the Kingdom of Saudi Arabia did not specify any clinical speciality or the number of respondents from either country. Eight of the studies scored $100 \%$ using the 
Mixed Methods Appraisal Tool and one study (Abudari et al., 2014) scored 75\% as the response rate was less than $60 \%$.

$<$ insert Table 1 here $>$

After analysis, three themes emerged from the review. Firstly, expatriate nurses saw themselves as powerless patient advocates and experienced frustration at the cultural limitations of their role. Secondly, the ability to provide culturally appropriate care was confounded by a quadriad communication structure of stakeholders with different languages and beliefs about death. Finally, expatriate nurses struggled with their emotions while negotiating culturally safe care in regard to religious practices. Each of these is more fully described in the following sections.

\subsection{Expatriate nurses view themselves as powerless patient advocates}

This theme related to the relationship between the nurses and physicians, with nurses' consistently positioning themselves as advocates for the patient but frustrated by limited opportunities to exercise their autonomy. Nurses positioned themselves as crucial to the provision of good end of life care (Abu-Ghori et al., 2016; Abudari et al., 2016; Alasiry et al., 2012) and to preparing the patient and family for death (Abudari et al., 2016). In several studies, nurses' reported that their opinions were not sought by family or physicians excluding them from end of life discussions (Abu-Ghori et al., 2016; Alosaimi et al., 2013; O'Neill et al., 2017). Nurses who were excluded from end of life decisions felt vulnerable when asked to explain care decisions to the patient and family (Abu-Ghori et al., 2016). Further, when nurses were not included in decisionmaking discussions, they reported feeling undervalued, using terms such as 'hired help' and sometimes, 'slaves' to describe how they felt (Alosaimi et al., 2013).

The study by Abu-Ghori et al. (2016) illustrated that nurses' sense of advocacy compelled them to encourage physicians to have further discussions with families to ensure clarity of understanding in relation to end of life decisions, such as 'do not resuscitate' orders. Nurses reported distress when they could see clear indicators that the person was approaching end of life 
but their exclusion from discussions meant that transition to end of life care was delayed. Nurses called this active treatment, provided when the person was approaching end of life, 'futile' care (Abudari et al., 2016; Halligan, 2006). 'Futile' care was most confronting for nurses in intensive care units when family directed continued life support in brain death and the cultural rules limited nurses' participation in any difficult conversations about withdrawing life support (Halligan, 2006; O'Neill et al., 2017).

In summary, nurses viewed themselves as the patient advocate and worked to negotiate their limited engagement in decision-making within a culture that excluded them from the discussions. The nurses wanted to exercise their professional skills but felt discouraged by the lack of opportunities to engage in the discussions related to withdrawing treatment.

\subsection{Nurses are hindered by the quadriad structure, language and differing beliefs about} communicating death.

The communication between the patient, physician, nurse and family emerges as complex and the term quadriad is used to describe this complexity. Although each stakeholder shared a common interest in the patient, the ways that individuals in the quadriad interacted could confound nurses attempts to clarify, communicate, and achieve a shared goal. Difficulty discussing and providing end of life care was a common theme in all studies. The value of culturally appropriate care was widely shared, but how it was enacted proved challenging.

Nurses worked within a rule bound, order centred process where communication about death, when and if it occurred, was considered the domain of the physician. In the intensive care setting, doctors would ask nurses to provide information about the family and their readiness to hear that the patient was unlikely to recover and advise on how the family might react (O'Neill et al., 2017). When communication about end of life was not delivered or not heard by families, nurses were confronted by family members seeking to understand the 'sudden' death of a family member (O'Neill et al., 2017). 
Nurses were often a conduit between the family and the physician and in some cases, the family drove decision-making. In one study (Abudari et al., 2016), nurses described the decisionmaking process at the end-of-life as family-directed. In another study, family-led decision-making was viewed negatively, as overruling the patient's autonomy, particularly when the family's decisions influenced medical decisions to continue what the nurses termed as 'futile' care (Alosaimi et al., 2013). Nurses working in the critical care setting were concerned that the family often 'dictated the care' without the patient's knowledge or contribution (Halligan, 2006).

Professionally, the nurses understood that giving the family 'bad news', such as indicating that death was near, was the domain of the physician (Abu-Ghori et al., 2016; Abudari et al., 2016; O'Neill et al., 2017) and reported feeling stressed when physician communication was ambiguous. For example, ambiguity existed when physicians continued to recommend aggressive treatment to a dying patient (Abudari et al., 2016) or not directly informing the family of the 'do not resuscitate' order (O'Neill et al., 2017) and using terms like 'critical' rather than 'dying' (Abu-Ghori et al., 2016; O'Neill et al., 2017). In other cases, nurses found themselves engaged in this ambiguity by undertaking 'placebo-type activities' of resuscitation to avoid family accusations of not providing adequate care (O'Neill et al., 2017). The nurses attributed these actions to the physicians' avoidance of death and the nurses' need to placate grieving relatives thus avoiding blame (O'Neill et al., 2017), illustrating the complexity of the communication quadriad.

Some nurses described physicians' unwillingness to give information to the family or tell the truth to patients and their family (Alasiry et al., 2012; O'Neill et al., 2017). In these cases, the nurses tried to find alternative ways to communicate that a patient was dying while facilitating a respectful and dignified death (O'Neill et al., 2017). Nurses in one study, set in a medical ward, directly attributed their focus on the patients' right to die and provision of continued family support to the physicians' lack of consensus around when end of life care begins (Abu-Ghori et al., 2016). Nurses in the study reported feeling excluded from conversations about end of life, attributing their 
exclusion from the 'do not resuscitate' decision-making process to their expatriate status and limited ability to speak Arabic (Abu-Ghori et al., 2016).

In the intensive care setting, nurses would use carefully chosen language and gestures to 'signal' to families that the death of a loved one was likely, to support families awareness of the possibility of imminent death (O'Neill et al., 2017). Yet, once a 'do not resuscitate' decision was made, nurses perceived physician withdrawal from care as abandonment and not consistent with their own valued provision of 'emotional support' (Abu-Ghori et al., 2016). In these cases, nurses felt compelled to bridge the gap in communication between the family and physician (Abu-Ghori et al., 2016).

In summary, nurses were continually managing and negotiating their relationships within the quadriad. The nurses were responsible to clarify family understanding of the end of life decisions and were concerned when families made decisions without consulting patients, or when physicians did not communicate about or initiate end of life care. When the nurses were excluded from conversations about end of life care due to their expatriate status or lack of language, or those conversations were not held, the nurses were surreptitious in their communication with the patient and families about end of life. Finally, when physicians did prescribe a 'do not resuscitate' order, their withdrawal from the care process was regarded by nurses as abandonment.

\subsection{Negotiating culturally safe care is emotionally challenging}

Nurses' experienced the negotiation of culturally safe care as a challenge rather than an achievement. A commonly reported challenge was nurse allocation in relation to gender allocation, when female patients would not accept care from a male nurse (Alosaimi \& Ahmad, 2016; AlYateem \& Al-Yateem, 2014; Halligan, 2006). Nurses reported delays in patient care delivery due to uneven workloads related to gendered nurse allocation (Alosaimi \& Ahmad, 2016; AlYateem \& Al-Yateem, 2014). 
Another source of challenge was to be culturally safe in their interactions with family. Nurses in some studies described family involvement negatively, using words such as 'interference'(Alosaimi et al., 2013), 'demanding'(Abudari et al., 2016) and 'hindering the delivery of nursing care'(Halligan, 2006).

Critical care nurses identified cultural practices, such as family frequently visiting in large numbers, bringing food and carpets, touching patients without regard of clinical status and visiting other patients as challenging behaviours (Halligan, 2006). Researchers concluded from another study in intensive care (Alasiry et al., 2012) that nurses acknowledged the need for additional skills to manage and support families and visitors, when visiting in large groups is common.

In terms of specific care practices, balancing clinical care with cultural practices was challenging. Balancing family's fear of opioid use and extended prayers with effective nursing care was recognised as important for cultural safety (Abudari et al., 2016; Alosaimi et al., 2013; AlYateem \& Al-Yateem, 2014; Halligan, 2006). For some nurses, prayer and fasting were considered to be disruptive, postponing treatments and placing patients at risk of adverse events such as falling while attending to ablution practices (Abudari et al., 2016). Other concerns related to missing medications and food (Alosaimi et al., 2013), requiring changes to meal and medication administration schedules and coordinating the delivery of food preferences (Alosaimi et al., 2013; AlYateem \& Al-Yateem, 2014). Several studies reported nurse resistance to positioning the patient's bed to face Mecca, on the grounds that this bed position limited their ability to administer oxygen or perform suctioning and therefore threatened patient safety (Abu-Ghori et al., 2016; Abudari et al., 2016; Alosaimi \& Ahmad, 2016; Alosaimi et al., 2013).

While several studies reported that nurses were challenged to balance cultural and clinical care, intensive care nurses (Alasiry et al., 2012), medical nurses (Abu-Ghori et al., 2016) and palliative care nurses (Abudari et al., 2016) demonstrated their respect to the patient and family through complying to as many cultural and religious requests as possible. For example, in critical 
care units, expatriate nurses played tapes of the Holy Qur'an when a patient was dying (O'Neill et al., 2017). However, in one study, AlYateem and Al-Yateem (2014) found that nurses efficiently managed and aligned their care with prayer times.

In summary, expatriate nurses' expectations of effective nursing care near end of life had to be continually negotiated in response to the patient and family enactments of culture and religion and the presence of large families. When nurses practiced in patient-focused ways, the enactments of culture and religion could align with nursing care. However, when nurses privileged clinical care over culture and religion, those nurses experienced emotional disruption, which was emotionally challenging.

\section{Discussion}

The reported accounts of expatriate nurses caring for a Muslim patient approaching end of life in their home country highlight the complex challenges and negotiations required to care for the dying patient within a different cultural framework. The small number of studies included in this review reflects the limited, but emerging, research on this topic.

The main theme was powerless patient advocacy. We conclude that nurses found themselves powerless and often in conflict with the patient, family or physicians particularly when their views differed culturally. Patient advocacy is a complex process of supporting patients emotionally and preserving their dignity (Munday et al., 2015; Negarandeh et al., 2006). Our analysis revealed that nurses in these studies struggled to find ways to express their view of appropriate clinical care with the physician, and this was compounded when excluded from decision-making.

Powerless patient advocacy may also be attributed to a cultural misunderstanding of patient autonomy. Autonomy from a Western model focuses on individual self-determination (Wilson et al., 2014) and refers to the patient's right to make free decisions about his or her health care based on their own values and beliefs (Rathor et al., 2016). As such, healthcare professionals' obligation to respect autonomy is not absolute. Rather, when autonomy is reduced to the single factor of an 
individual's choice, its universal validity can be challenged (Rathor et al., 2016). Respect for cultural diversity requires consideration of other values such as supporting family integrity and reducing distress for patients can be more important near end of life (Barclay et al., 2007).

The concept of autonomy in the Islamic model comes from divine origin and is reserved for the principle of public benefit, where the collective interest takes precedence over that of individual autonomy, and the family often remains the important subject for the patient's decisions in difficult situations (Rathor et al., 2016). When nurses were unable to adapt to the cultural aspect of providing nursing care in a Muslim country, they perceived a lack of autonomy and a sense of frustration. Those nurses who perceived cultural practices as interfering, may be undergoing role morality stress due to difficulty separating personal and professional values (Hanna, 2004), where professional values of care delivery may be clinically or person-focused.

The potential for miscommunication within a quadriad communication structure was significant. The nurses from these studies understood that the role of discussing end of life and prognosis remains with the physician but sought opportunities for engagement in that discussion. Nurses reported that families were not only involved in discussions but also directed decisions. The diversity of family responses, patient preferences, and variable capacities of physicians and nurses to hold discussions about death and dying make the process of communicating complex (Barclay et al., 2007). Consistent with our findings, health care professionals in other settings also experienced difficulty and barriers to effective communication at the end of life when working with families, cultural differences, and prognostic uncertainty (Anselm et al., 2005; Barclay et al., 2007; Randhawa et al., 2003). Decision making at the end of life is extremely difficult and cultural differences create even greater challenges for health care professionals balancing their personal and professional views on end of life care within different cultural contexts (Barclay et al., 2007). This is an area for further research. 
Nurses in the reviewed studies described being present during the dying process, developing trusting relationships with patients and families, and answering questions regarding prognosis and treatment. However, due to language barriers and local cultural practices, the nurses reported feeling excluded from decisions about end of life care. Evidence about effective communication strategies near end of life suggest that disclosing bad news or truth telling in a nonverbal manner as is preferred from some cultures who expect answers to be more euphemistic and indirect (Barclay et al., 2007).

The balance between clinical and cultural care emerges as an important skill for expatriate nurses, but it was also a source of stress, due to insufficient knowledge of the cultural requirements and ability to provide cultural care. Opportunities for nurses to discuss their experiences with other nurses and cultural experts, on a case-by-case basis could support reflection on practice and identify areas for greater alignment between culture and clinical goals of care.

Expatriate nurses found negotiating their work culture within a Muslim country emotionally challenging. Some nurses showed commitment and sensitivity to patients' culture and faith although this was sometimes challenging to enact. Providing culturally competent end of life care is recognised as difficult (Doolen \& York, 2007), especially for expatriate nurses (Almutairi \& McCarthy, 2012). For some nurses, the interruptions and continual negotiations with patients and family in relation to cultural practices disrupted the provision of care. It is notable that the nurses in these studies did not discuss how their own cultural norms influenced their interactions, indicating a possible lack of self-awareness. A continuing education program for cultural awareness may support nurses to align their perspectives on practice priorities with the cultural context.

Nurses' experiences of cultural clashes and conflicts often stems from a lack of cultural knowledge (Boi, 2000; Cioffi, 2005; Hoye \& Severinsson, 2008; Kirkham, 1998; Murphy \& Macleod Clark, 1993; Shanahan \& Brayshaw, 1995; Vydelingum, 2006). The lack of awareness of patients' specific health beliefs and religious requirements could affect the nurses' ability to provide 
holistic care (Almutairi, 2012). One way to support expatriate nurses in a Muslim country may be to introduce arriving nurses to a local nursing model of care. For example, the Crescent of Care Nursing Model of Care provides a holistic approach for Arab Muslim nurses caring for Arab Muslim patients in the Middle East region and focuses on the spiritual, cultural, psychosocial, interpersonal and clinical caring needs of Arab Muslims. It was developed in the Kingdom of Saudi Arabia and is reportedly used to guide nursing and nurse curricula and serves as a general guide for the care of Muslim patients in other settings (Lovering, 2012). Further exploration of this model of care, particularly to support expatriate nurses, is recommended.

Atkinson (2015) in her qualitative study of Muslim nurses in Kuwait, asserts it may not be possible to completely separate the influence of religion from that of culture. In her study of Islamic values on nurses role and their practice, Muslim nurses have described their work as spiritual practice and their patients as spiritual beings (Atkinson, 2015), and answered cultural questions with religious content (Mebrouk, 2008). Halligan (2006) asserts that the concept of caring has been examined extensively in the context of Western cultures, and nurses in the West tend to make a distinction between physical and spiritual care (Atkinson, 2015). Further, spiritual needs may be confused with psychological needs or given to the care of an assigned pastoral/spiritual advisor (Lemmer, 2005). Improving cultural, as well as spiritual, competence ensures that health professionals can provide optimal end of life care to patients from all walks of life and all cultural backgrounds (Givler \& Maani-Fogelman, 2018).

\section{Limitations}

This review only included articles in English, excluding any studies published in other languages. The small number of studies and participants means that this may not represent all nurses' experiences and as such the findings must be viewed as tentative.

\section{Implications for Practice, Research and Education}


Through this review, we conclude that while expatriate nurses endeavour to learn how to navigate culture, religion and language while 'on the job', finding themselves in conflict with other health professionals, patient and families can be challenging and emotionally stressful work.

Expatriate nurses from non-Muslim countries will need to understand the influence that Islamic culture and religion have on end of life care. We recommend a research program focused on those clinicians who are integrating culture, spirituality and clinical care to identify professional strategies that work and can potentially reduce emotional distress for expatriate nurses.

From an organisational perspective, we suggest that educational programs that are focused on cultural awareness and personal experiences are required. Opportunities for nurses to reflect on their practice and explore their experiences of cultural and professional conflict could be achieved through peer discussions known as 'learning circles'. Learning circles focus on individual cases or particular topics (Walker et al., 2011). They could provide an opportunity for participants to reflect on and improve their practice in collaboration with cultural experts.

Finally, we recommend introducing expatriate nurses to local models of nursing care, which have culture and spirituality as key elements of care. The potential for improving perceptions and building relationships and increasing cultural knowledge would likely reduce the stress nurses reported in these studies.

\section{Conclusion}

We have found in this literature review that working within a country and health system with a culture, language and religion, that is different from your own is stressful, particularly during end of life care. Cultural differences influenced patient advocacy, communication and in some cases, nurses' emotional response to these differences. Opportunities to discuss within health care teams the nuanced moral dilemmas that arise for expatriate nurses in a Muslim country may offer one strategy to reduce their emotional distress experienced while they negotiate values that differ from their own. 
Importantly, if countries such as Kingdom of Saudi Arabia continue to rely on expatriate nurses, cultural care education and implementation of appropriate models of care would enable nonMuslim nurses to be fully cognisant about and sympathetic to Islamic practices that are necessary for providing competent, culturally sensitive holistic end of life care to Muslim patients.

\section{Conflict of Interest}

All authors declare that they have no conflict of interest.

\section{Funding}

This research received no specific grant from any funding agency. 


\section{References}

Abel, J., \& Kellehear, A. (2016). Palliative care reimagined: A needed shift. BMJ Supportive \& Palliative Care, 6(1), 21. doi:http://dx.doi.org.libraryproxy.griffith.edu.au/10.1136/bmjspcare-2015-001009

Aboshaiqah, A. (2016). Strategies to address the nursing shortage in Saudi Arabia. International Nursing Review, 63, 499-506.

Aboshaiqah, A., Tumala, R., Inocian, E., Almutairi, A., \& Atallah, M. (2017). Enhancing Culturally Competent Nursing Care in Saudi Arabia. Journal of Cultural Diversity, 24(1), 20-26.

Abu-Ghori, I. K., Bodrick, M. M., Hussain, R., \& Rassool, G. H. (2016). Nurses' involvement in end-of-life care of patients after a do not resuscitate decision on general medical units in Saudi Arabia. Intensive Crit Care Nurs, 33, 21-29. doi:10.1016/j.iccn.2015.10.002

Abudari, G., Hazeim, H., \& Ginete, G. (2016). Caring for terminally ill Muslim patients: Lived experiences of non-Muslim nurses. PALLIATIVE \& SUPPORTIVE CARE, 14(6), 599-611. doi:10.1017/S1478951516000249

Abudari, G., Zahreddine, H., Hazeim, H., Al Assi, M., \& Emara, S. (2014). Knowledge of and attitudes towards palliative care among multinational nurses in Saudi Arabia. International Journal of Palliative Nursing, 20(9), 435-441.

Al-Yateem, N., AlYateem, S., \& Rossiter, R. (2015). Cultural and Religious Educational Needs of Overseas Nurses Working in the Kingdom of Saudi Arabia and the United Arab Emirates. Holistic Nursing Practice, 29(4), 205-215. doi:http://dx.doi.org/10.1097/HNP.0000000000000095

Alasiry, S., Alshehri, H., Medin, J., \& Hagelin, C. L. (2012). Nurses' Experiences of Providing Palliative Care in an Intensive Care Unit in Saudi Arabia. Middle East Journal of Nursing, 19-30.

Almutairi, A. (2012). A case study examination of the influence of cultural diversity in the multicultural nursing workforce on the quality of care and patient safety in a Saudi Arabian hospital. (Doctor of Health Science), Queensland University of Technology, Brisbane, Australia.

Almutairi, A., \& McCarthy, A. (2012). A multicultural nursing workforce and cultural perspectives in Saudi Arabia : an overview. The Health, 3(3), 71-74.

Almutairi, A., \& Rodney, P. (2013). Critical Cultural Competence for Culturally Diverse Workforces Toward Equitable and Peaceful Health Care. Advances in Nursing Science, 36(3), 200-212. doi: http://dx.doi.org/10.1097/ANS.0b013e31829edd51

Alosaimi, D., \& Ahmad, M. (2016). The Challenges of Cultural Competency Among Expatriate Nurses Working in Kingdom Saudi Arabia. Research and Theory for Nursing Practice, 30(4), 302-319.

Alosaimi, D., Dyson, S., \& Anthony, D. (2013). A Phenomenological Study Of Non-Muslim Nurses Expereince Of Caring For Muslim Patients In Saudi Arabia. International Journal of Arts \& Sciences, 6(2), 637-646.

AlYateem, S., \& Al-Yateem, N. (2014). The Experience of Overseas Nurses Caring For Muslim Patients In Kingdom of Saudi Arabia and UAE: A Qualitative Study International Journal of Research in Nursing, 17-24. doi:http://dx.doi.org/10.3844/ijrnsp.2014.17.24

Anselm, A. H., Palda, V., Guest, C. B., McLean, R. F., Vachon, M. L., Kelner, M., \& LamMcCulloch, J. (2005). Barriers to communication regarding end-of-life care: perspectives of care providers. J Crit Care, 20(3), 214-223. doi:10.1016/j.jcrc.2005.05.012

Ashby, M. (2016). How we die: a view from palliative care. QUT Law Review., 16(1), 5-21. doi:http://dx.doi.org/10.5204/qutlr.v16il.619

Atkinson, C. (2015). Islamic Values and Nursing Practice in Kuwait. J Holist Nurs, 33(3), 195-204. doi:10.1177/0898010114564682 
Barclay, J. S., Blackhall, L. J., \& Tulsky, J. A. (2007). Communication Strategies and Cultural Issues in the Delivery of Bad News. Journal of Palliative Medicine, 958-977. doi:http//:dx.doi.org/10.1089/jpm.2007.9929

Bloomer, M. J., \& Al-Mutair, A. (2013). Ensuring cultural sensitivity for Muslim patients in the Australian ICU: Considerations for care. AUSTRALIAN CRITICAL CARE, 26(4), 193-196. doi:10.1016/j.aucc.2013.04.003

Boi, S. (2000). Nurses' experiences in caring for patients from different cultural backgrounds. Nursing Times Research, 5(5), 382 - 389.

Bucknall, T. K., Hutchinson, A. M., Botti, M., McTier, L., Rawson, H., Hewitt, N. A., . . . Chaboyer, W. (2016). Engaging patients and families in communication across transitions of care: an integrative review protocol. $J$ Adv Nurs, 72(7), 1689-1700. doi:10.1111/jan.12953

Cashavelly, B. J., Donelan, K., Binda, K. D., Mailhot, J. R., Clair-Hayes, K. A., \& Maramaldi, P. (2008). The forgotten team member:meeting the needs of oncology support staff. Oncologist, 13(5), 530-538.

Cioffi, J. (2005). Nurses' experiences of caring for culturally diverse patients in an acute care setting. Contemp Nurse, 20(1), 78-86.

Commonwealth of Australia. (2010). National Palliative Care Strategy 2010 Supporting Australians to Live Well at the End of Life. Australia: Commonwealth of Australia Retrieved from http://www.ag.gov.au/cca.

Doolen, J., \& York, N. L. (2007). Cultural Differences with end-of-life care in the critical care unit. . Dimensions of Critical Care Nursing, 26(5), 194-198. doi:DOI: 10.1097/01.DCC.0000286822.04238.df

Dréano-Hartz, S., Rhondali, W., Ledoux, M., Ruer, M., Berthiller, J., Schott, A., . . Filbert, M. (2015). Burnout among physicians in palliative care: Impact of clinical settings. PALLIATIVE \& SUPPORTIVE CARE, 4(4), 402-410. doi:DOI:10.1017/S1478951515000991

Eagar, K., Watters, P., Currow, D. C., Aoun, S. M., \& Yates, P. (2010). The Australian Palliative Care Outcomes Collaboration (PCOC)--measuring the quality and outcomes of palliative care on a routine basis. Aust Health Rev, 34(2), 186-192. doi:10.1071/AH08718

El-Amouri, S., \& O'Neill, S. (2011). Supporting cross-cultural communication and culturally competent care in the linguistically and culturally diverse hospital settings of UAE. Contemp Nurse, 39(2), 240-255. doi:10.5172/conu.2011.240

El-Haddad, M. (2006). Nursing in the United Arab Emirates: an historical background. Int Nurs Rev, 53(4), 284-289. doi:10.1111/j.1466-7657.2006.00497.x

Fairbrother, C. A., \& Paice, J. A. (2005). Life's final journey: the oncology nurse's role. CLINICAL JOURNAL OF ONCOLOGY NURSING, 9(5), 575-579.

Garner, K. K., Goodwin, J. A., McSweeney, J. C., \& Kirchner, J. E. (2013). Nurse executives' perceptions of end-of-life-care provided in hospitals. Journal of Pain and Symptom Management, 45(2), 235-242.

Gatrad, R., \& Sheikh, A. (2002). Palliative care for Muslims and issues after death. Int J Palliat Nurs, 8(12), 594-597. doi:10.12968/ijpn.2002.8.12.10977

Givler, A., \& Maani-Fogelman, P. A. (2018). Cultural Competence in Pain and Palliative Care Retrieved from https://www.ncbi.nlm.nih.gov/books/NBK493154/

Greenberg, D., \& Haddonfield, N. (2007). Gendered Expressions of Grief. Journal of Religion \& Society, 9.

Halligan, P. (2006). Caring for patients of Islamic denomination: Critical care nurses' experiences in Saudi Arabia. J Clin Nurs, 15(12), 1565-1573. doi:10.1111/j.1365-2702.2005.01525.x

Hanna, D. (2004). Moral Distress: The State of the Science. Research and Theory for Nursing Practice: An International Journal, 18(1), 73-93. 
Hawkins, J. (2015). 2015 health care outlook Middle East. Retrieved from https://www2.deloitte.com/content/dam/Deloitte/global/Documents/Life-Sciences-HealthCare/gx-1shc-2015-health-care-outlook-middle-east.pdf

Hoye, S., \& Severinsson, E. (2008). Intensive care nurses' encounters with multicultural families in Norway: An exploratory study. Intensive \& Critical Care Nursing, 24, 338 - 348. doi:10.1016/j.iccn.2008.03.07

Kirkham, S. R. (1998). Nurses' Descriptions of Caring for Culturally Diverse Clients. Clinical Nursing Research, 7(2), 125 - 146.

Leininger, M. (1988). Leininger's theory of nursing: cultural care diversity and universality. Nurs Sci Q, 1(4), 152-160. doi:10.1177/089431848800100408

Lemmer, C. M. (2005). Recognizing and caring for spiritual needs of clients. J Holist Nurs, 23(3), 310-322. doi:10.1177/0898010105277652

Liberati, A., Altman, D., Tetzlaff, J., Mulrow, C., Gøtzsche, P., Ioannidis, J., . . Moher, D. (2009). The PRISMA statement for reporting systematic reviews and meta-analyses of studies that evaluate healthcare interventions: explanation and elaboration. Journal of Clinical Epidemiology, 62, e1-e34. doi:10.1016/jclinepi.2009.06.006

Lovering, S. (2012). The Crescentof Care: a nursing model to guide the care of Arab Muslim patients. Diveristy and Equality in Health Care, 9, 171-178.

Lu, Y. H., Guo, R. X., \& Liu, L. (2011). Oncology nurses' identification with their responsibilities and negative experience in caring for dying cancer patients. Chinese Nursing Management, 11(2), 84-86.

Mebrouk, J. (2008). Perception of nursing care: Views of Saudi Arabian female nurses. Contemporary Nurse, 28(1), 149 - 161.

Moher, D., Liberati, A., Tetzlaff, J., Altman, D. G., \& Group, P. (2009). Preferred reporting items for systematic reviews and meta-analyses: the PRISMA statement. BMJ, 339, b2535. doi:10.1136/bmj.b2535

Munday, J., Kynoch, K., \& Hines, S. (2015). Nurses' experience of advocacy in the perioperatitve department. A systematic review. JBI Database of Systematic Reviews \& Implementation Reports, 13, 146-189.

Murphy, K., \& Macleod Clark, J. (1993). Nurses' experiences of caring for ethnic-minority clients. Journal of Advanced Nursing, 18(3), 442 - 450.

Negarandeh, R., Oskouie, F., Ahmadi, F., Nikravesh, M., \& Hallberg, I. R. (2006). Patient advocacy: barriers and facilitators. BMC Nursing, 5(3).

O'Neill, C. S., Yaqoob, M., Faraj, S., \& O'Neill, C. L. (2017). Nurses' Care Practices at the End of Life in Intensive Care Units in Bahrain. Nursing Ethics, 24(8), 950-961. doi:doi:10.1177/0969733016629771

Peters, L., Cant, R., Payne, S., O'Connor, M., McDermott, F., Hood, K., . . Shimoinaba, K. (2013). How Death Anxiety Impacts Nurses' Caring for Patients at the End of Life: A review of Literature. The Open Nursing Journal, 7, 14-21.

Peterson, J., Johnson, M., Halvorsen, B., Apmann, L., Chang, P. C., Kershek, S., . . Pincon, D. (2010). What is it so stressful about caring for a dying patient? A qualitative study of nurses' experiences International Journal of Palliative Nursing, 16(4), 181-187.

Pluye, P., Gagnon, M. P., Griffiths, F., \& Johnson-Lafleur, J. (2009). A scoring system for appraising mixed methods research, and concomitantly appraising qualitative, quantitative and mixed methods primary studies in Mixed Studies Reviews. Int J Nurs Stud, 46(4), 529546. doi:10.1016/j.ijnurstu.2009.01.009

Pluye, P., \& Hong, Q. N. (2014). Combining the power of stories and the power of numbers: mixed methods research and mixed studies reviews. Annu Rev Public Health, 35, 29-45.

doi:10.1146/annurev-publhealth-032013-182440 
Polit, D. F., \& Beck, C. T. (2017). Nursing Research: generating and assessing evidence for nursing practice. (10th ed.). Philadelphia: Wolters Kluwer Health.

Portoghese, I., Galletta, M., Coppola, R. C., Finco, G., \& Campagna, M. (2014). Burnout and workload among health care workers: the moderating role of job control. Saf Health Work, 5(3), 152-157. doi:10.1016/j.shaw.2014.05.004

Randhawa, G., Owens, A., Fitches, R., \& Khan, Z. (2003). Communication in the development of culturally competent palliative care services in the UK: a case study. Int J Palliat Nurs, 9(1), 24-31. doi:10.12968/ijpn.2003.9.1.11042

Rathor, M. Y., Azarisman Shah, M. S., \& Hasmoni, M. H. (2016). Is Autonomy a Universal Value of Human Existence? Scope of Autonomy in Medical Practice: A Comparative Study between Western Medical Ethics and Islamic Medical Ethics. International Medical Journal of Malaysia, 15(1), 81-88.

Reinhard, S. C., Given, B., Petlick, N. H., \& Bemis, A. (2008). Supporting Family Caregivers in Providing Care. In R. G. Hughes (Ed.), Patient Safety and Quality: An Evidence-Based Handbook for Nurses. Rockville (MD): Agency for Healthcare Research and Quality (US).

Ribeiro, V. F., Filho, C. F., Valenti, V. E., Ferreira, M., de Abreu, L. C. d. C., T. D., Xavier, V., . . . Ferreira, C. (2014). Prevalence of burnout syndrome in clinical nurses at a hospital of excellence. International Archives of Medicine, 7, 22.

Salman, K., \& Zoucha, R. (2010). Considering Faith Within Culture When Caring for the Terminally Ill Muslim Patient and Family. Journal of Hospice and Palliative Care, 12(3), 156-163.

Shanahan, M., \& Brayshaw, D. L. (1995). Are nurses aware of the differing health care needs of Vietnamese patients? Journal of Advanced Nursing, 22, 456 - 464

Somerville, J. (2007). The paradox of palliative care nursing across cultural boundaries. Int J Palliat Nurs, 13(12), 580-587. doi:10.12968/ijpn.2007.13.12.27886

Thurston, J., \& Waterworth, S. (2012). 'Making sense' nurses' experiences of changing practice for dying patients in New Zealand. International Journal of Palliative Nursing, 18(10), 500507.

Vydelingum, V. (2006). Nurses' experiences of caring for South Asian minority ethnic patients in a general hospital in England. Nurs Inq, 13(1), 23-32. doi:10.1111/j.1440-1800.2006.00304.x

Walker, R., Henderson, A., Cooke, M., \& Creedy, D. (2011). Impact of a learning circle intervention across academic and service contexts on developing a learning culture. Nurse Educ Today, 31(4), 378-382. doi:10.1016/j.nedt.2010.07.010

Whittemore, R., \& Knafl, K. (2005). The integrative review: updated methodology. J Adv Nurs, 52(5), 546-553. doi:10.1111/j.1365-2648.2005.03621.x

Wilson, F., Ingleton, C., Gott, M., \& Gardiner, C. (2014). Autonomy and choice in palliative care: time for a new model? Journal of Advanced Nursing, 70(5), 1020-1029. doi:10.1111/jan.12267

Wu, S. Y., Li, H. Y., Tian, J., Zhu, W., Li, J., \& Wang, X. R. (2011). Health-related quality of life and its main related factors among nurses in China. Industrial Health, 49(2), 158-165. 
